\title{
Comparison of duration of maternal hospital stay in conservative $\&$ aggressive management of Preterm prelabor rupture of membranes(PPROM) in Indian women at 34-36 weeks gestation.
}

\author{
Shachi Shukla ${ }^{1}$, Kshitij Sharma ${ }^{2}$ \\ ${ }^{I}$ Resident, Department of Obstetrics \&Gynaecology, SMS Medical College, Jaipur(Rajasthan), India. \\ ${ }^{2}$ Department of civil engineering, Indian Institute of Technology, Kanpur, (Uttar Pradesh), India
}

\begin{abstract}
:
Aim:To compare the conservative and aggressive management of Preterm prelabor rupture of membranes (PPROM) in Indian women at 34-36 weeks gestation in terms of duration of their hospital stay.

Materials \& Method:194 pregnant women with PPROM at gestational age 34-36 weeks admitted in labour ward were included in the study conducted from 2009 -2010 in department of Obstetrics \& Gynaecology, SMS Medical College, India. Random allocation to conservative \& aggressive management group was done by offering Chit Box Method assigning 97 cases in each group.

In conservative management cases were hospitalized\& provided bed rest, Daily fetal monitoring, maternal vitals monitoring, Oral tab erythromycin $500 \mathrm{mg}$ t.d.s for 7 days, Daily WBC count and C - reactive protein estimation. Expectant management was abandoned if there was clinical evidence of labour, infection or fetal distress.

In aggressive management induction of labour was done by Tab Misoprostol $25 \mu \mathrm{g}$ orally, at 4-6 hour intervals, for a maximum of 5 doses. Caesarean delivery was performed for standard obstetrical indication and for failed induction.

After delivery neonatal care was provided by neonatologist

Results: Out of total 97 cases, in aggressive management, majority 73 (75.26\%) cases had duration $\leq 5$ days, $19(19.59 \%)$ up to 6-10 days and $5(5.15 \%)$ cases up to $11+$ days. Similarly out of total 97 cases in conservative management, majority $46(47.42 \%)$ cases had duration $\leq 5$ days, 33 (34.02\%) up to 6-10 days and 18 (18.56\%) cases up to $11+$ days.

The mean duration of hospital stay of mother in aggressive management were $4.10 \pm 3.10$ days and in conservative management it is $7.21 \pm 3.36$ days.

Conclusion:Majority of cases in both aggressive and conservative management group had duration of hospital stay of $\leq 5$ days. But the total duration of hospital stay of mother were much higher in conservative management group in present study.

Thus mean duration of hospital stay of mother were much higher in conservative management group as compared to aggressive management.
\end{abstract}

Keywords-PPROM, conservative, aggressive, hospital stay.

\section{Introduction}

Preterm prelabor rupture of fetal membranes (PPROM) is defined as rupture of fetal membranes prior to the onset of labour at less than 37 weeks of gestation. The fetal membranes serve as a barrier to ascending infection. Once the membranes rupture, both the mother and foetus are at risk of infection and of other complications. Most women with PPROM go into spontaneous labour within 24 hours of rupturing their membranes, but $6 \%$ of women will not be in spontaneous labour within 96 hours. However earlier in gestation the rupture occurs, the less likely that the onset of labour will be within a specified time period. PPROM occurs in 2 to $3 \%$ of all pregnancies and proceeds $1 / 3$ of preterm births and 18 to $20 \%$ of perinatal deaths. PPROM is largely a clinical diagnosis characterised by a history of watery vaginal discharge. Prolonged rupture of membrane (PROM) is an important risk factor for chorioamnionitis.Chorioamnionitis is a common complication of pregnancy associated with significant maternal, perinatal, and long-term adverse outcomes. Adverse maternal outcomes include postpartum infections and sepsis while adverse infant outcomes include stillbirth, premature birth, neonatal sepsis, chronic lung disease and brain injury leading to cerebral palsy and other neurodevelopmental disabilities

Conservative versus aggressive management of PPROM is amongst the most controversial issue and still remains a major dilemma to the obstetricians. Present study is intended to compare the conservative and aggressive management of PPROM at 34-36 weeks gestation in terms of duration of hospital stay. 


\section{Material And Methods}

This prospective randomized clinical trial study was conducted in the Department of Obstetrics and Gynaecology, SMS Medical College, Jaipur during the year 2009-2010.194 pregnant women with PPROM at gestational age 34-36 weeks admitted in labour ward were recruited in the present study. Written informed consent by each subject was sought before the study. Random allocation to both the management groups was done by offering Chit Box Method to eligible patients assigning 97 cases to each group. Inclusion Criteria were pregnant women with PPROM at 34-36 weeks of gestation. Exclusion Criteria were cases requiring delivery viz. labour, infection, fetal distress, lethal fetal anomalies / fetal demise, Maternal medical disorders, Non-vertex presentation, Antepartum haemorrhage, absolute indication for caesarean section.

PPROM was confirmed by sterile speculum examination, single digital examination, USG for AFI. Eligible women were assigned to either the conservative management or aggressive management group.

1. Group A (Conservative Management) - 97 cases

2. Group B (Aggressive Management) - 97 cases

Women of both groups were subjected to general physical examination, per abdomen examination

Per speculum / Pervaginal examination, sterile single digital examination to exclude occult cord prolapse and to assess cervical score.

Conservative management consists of Hospitalized bed rest, Daily Fetal Movement count, Intermittent FHS auscultation, NST, Maternal vitals monitoring every 8 hourly, Digital vaginal examination - Prohibited, Oral antibiotics - Tab Erythromycin $500 \mathrm{mg}$ TDS for 7 days, Daily leukocyte count and CRP estimation. Expectant management was abandoned if there is clinical evidence of labour, infection or fetal distress.

In aggressive management group induction of labour was done by Tab Misoprostol $25 \mu \mathrm{g}$ orally, at 4-6 hour intervals, for a maximum of 5 doses. (ACOG Recommendation) or caesarean delivery was performed for standard obstetrical indication and for failed induction.

After Delivery neonatal care was provided by neonatologist. Neonatal outcome variables of interest e.g. RDS, neonatal sepsis and other neonatal complications were noted.

All the data was entered in Excel Sheet and the data was analyzed statistically using XL Stat and Statcal Software. Quantitative data was summarized in the form of Mean \pm SD and the difference in mean value of both the groups were analyzed using Student's ' $t$ ' test. Qualitative data was summarized in the form of proportions and difference in proportion was analyzed using Chi Square test. All the statistical analysis was done at $95 \%$ confidence level and $80 \%$ power.

\section{Results}

Out of total 97 cases, in aggressive management, majority $73(75.26 \%)$ cases had duration $\leq 5$ days, 19 (19.59\%) up to 6-10 days and $5(5.15 \%)$ cases up to $11+$ days. Similarly out of total 97 cases in conservative management, majority $46(47.42 \%)$ cases had duration $\leq 5$ days, $33(34.02 \%)$ up to $6-10$ days and $18(18.56 \%)$ cases up to $11+$ days as shown in table-1 and fig. 1 .

Thus majority of cases in both aggressive and conservative management group had duration of hospital stay of $\leq 5$ days. But the total duration of hospital stay of mother were much higher in conservative management group in present study.

The mean duration of hospital stay of mother in aggressive management were $4.10 \pm 3.10$ days and in conservative management it is $7.21 \pm 3.36$ days.

\section{Discussion}

Present study shows that mean duration of hospital stay of mother were much higher in conservative management group as compared to aggressive management. This is similar to the findings of Brien M Mercer et $\mathrm{al}^{1}$ which shows immediate induction of labor reduces the duration of hospitalization and infection in both mothers and neonates.

This is also in accordance to the study of Eliezer Shalevet $\mathrm{al}^{2}$ showed that women with expectant management had longer hospital stay than active management (6 days v/s 5 days).

This is also in concordance to the study of Naef RW et $\mathrm{al}^{3}$ which showed that maternal hospital stay $(5.2+/-6.8$ days vs $2.6+/-1.6$ days, $\mathrm{p}=0.006)$ was significantly longer in the expectant management group compared to aggressive management group.

The results are also similar to the findings ofHartling Let $\mathrm{al}^{4}$ which showed that maternal length of stay was significantly shorter for the intentional delivery group (WMD - 1.39 days, 95\%, CI - $2.03-0.75$ ).

This is also in accordance to the findings of Buchanan SL et al ${ }^{5}$ which showed that there was significant reduction of duration of maternal hospital stay in early delivery group (MP - 1.13 days, 95\%, CI 1.75 to 0.51 days) as compared to expectant management group. 


\section{Conclusion}

Majority of cases in both aggressive and conservative management group had duration of hospital stay of $\leq 5$ days. But the total duration of hospital stay of mother were much higher in conservative management group in present study.

Thus mean duration of hospital stay of mother were much higher in conservative management group as compared to aggressive management.

\section{References}

[1]. Mercer BM, Crocker LG, Boe NM, SibaiBM.Induction versus expectant management in premature rupture of the membranes with mature amniotic fluid at 32 to 36 weeks: a randomized trial.Am J Obstet Gynecol. 1993 Oct;169(4):775-82.

[2]. Shalev E, Peleg D, Eliyahu S, Nahum Z.Comparison of 12- and 72-hour expectant management of premature rupture of membranes in term pregnancies. Obstet Gynecol. 1995 May;85(5 Pt 1):766-8.

[3]. Naef RW 3rd, Allbert JR, Ross EL, Weber BM, Martin RW, Morrison JC.Premature rupture of membranes at 34 to 37 weeks' gestation: aggressive versus conservative management.Am J Obstet Gynecol. 1998 Jan;178(1 Pt 1):126-30.

[4]. Hartling L, Chari R, Friesen C, Vandermeer B, Lacaze-Masmonteil T.A systematic review of intentional delivery in women with preterm prelabor rupture of membranes. J Matern Fetal Neonatal Med. 2006 Mar;19(3):177-87.

[5]. Buchanan SL, Crowther CA, Levett KM, Middleton P, Morris J.Planned early birth versus expectant management for women with preterm prelabour rupture of membranes prior to 37 weeks' gestation for improving pregnancy outcome. Cochrane Database Syst Rev. 2010 Mar 17;(3):CD004735. doi: 10.1002/14651858.CD004735.pub3.

Table - 1: Duration of Hospital Stay of Mother in Aggressive and Conservative Management

\begin{tabular}{|c|c|c|c|c|}
\hline \multirow{2}{*}{$\begin{array}{c}\text { Duration of Hospital } \\
\text { Stay }\end{array}$} & \multicolumn{2}{|c|}{ Aggressive Management } & \multicolumn{2}{|c|}{ Conservative Management } \\
\hline & No. of Cases & Percentage (\%) & No. of Cases & Percentage (\%) \\
\hline$\leq 5$ & 73 & 75.26 & 46 & 47.72 \\
\hline $6-10$ & 19 & 19.59 & 33 & 34.02 \\
\hline $11+$ & 5 & 5.15 & 18 & 18.56 \\
\hline Mean \pm SD & \multicolumn{2}{|c|}{$4.10 \pm 3.10$} & \multicolumn{2}{|c|}{$7.21 \pm 3.36$} \\
\hline Total & 97 & 100.00 & 97 & 100.00 \\
\hline
\end{tabular}

Figure-1

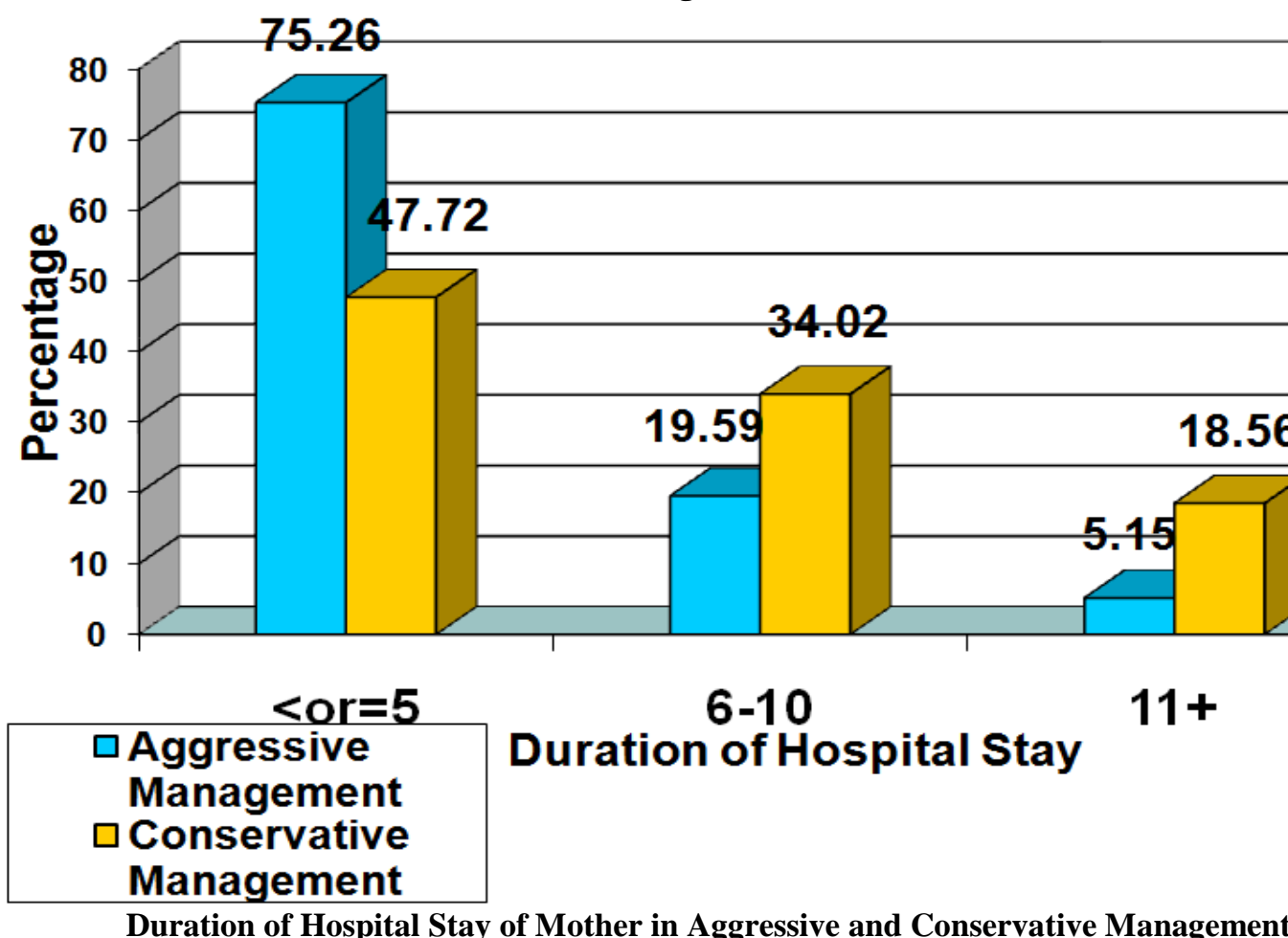

